\title{
Meta-Analysis of Randomized and Controlled Treatment Trials for Achalasia
}

\author{
Lan Wang $\cdot$ You-Ming Li $\cdot$ Lan Li
}

Published online: 18 June 2009

(C) Springer Science+Business Media, LLC 2009

\section{Erratum to: Dig Dis Sci}

\section{DOI 10.1007/s10620-008-0637-8}

The article was published with several citation errors in Table 1. The corrected Table 1 appears below.

The online version of the original article can be found under doi: 10.1007/s10620-008-0637-8.

L. Wang · Y.-M. Li $(\bowtie) \cdot$ L. Li Department of Gastroenterology, The First Affiliated Hospital, Medical College, Zhejiang University, No.79 Qingchun Road, Hangzhou 310003, People's Republic of China

e-mail: zlym@zju.edu.cn 
Table 1 Characteristics of 16 studies included in our meta-analysis of different treatment methods for achalasia

\begin{tabular}{|c|c|c|c|c|c|c|c|c|}
\hline \multirow{2}{*}{$\begin{array}{l}\text { The mode of } \\
\text { comparison }\end{array}$} & \multirow[t]{2}{*}{ Reference } & \multirow{2}{*}{$\begin{array}{l}\text { Sample size, } \\
\text { mode of } \\
\text { treatment }\end{array}$} & \multirow{2}{*}{$\begin{array}{l}\text { Age, mean } \\
\text { (range), year }\end{array}$} & \multirow{2}{*}{$\begin{array}{l}\text { Sex } \\
(\% \text { male })\end{array}$} & \multirow{2}{*}{$\begin{array}{l}\text { Duration of } \\
\text { symptoms, } \\
\text { mean (range), } \\
\text { month }\end{array}$} & Sympto & ores & \multirow{2}{*}{$\begin{array}{l}\text { Median follow-up } \\
\text { mean (range), } \\
\text { month }\end{array}$} \\
\hline & & & & & & Before & After & \\
\hline
\end{tabular}

Non-sugery methods: differences treatment methods compared with pneumatic dilation

\begin{tabular}{|c|c|c|c|c|c|c|c|}
\hline \multirow[t]{2}{*}{ Coccia et al. [14] } & $16 \mathrm{PD}$ & $49.5(18-82)$ & 56.25 & $49(6-96)$ & NA & & $21(12-36)$ \\
\hline & 14 nifedipine & $51(17-83)$ & 57.14 & $48(6-84)$ & NA & & \\
\hline \multirow[t]{2}{*}{ Vaezi et al. [15] } & 24 BoTx & $57(44-69)$ & 41.47 & NA & $11(9-13)$ & $2(0-2.5)$ & NA \\
\hline & $24 \mathrm{PD}$ & $56(37-70)$ & 29.17 & NA & $10(9-12)$ & $3(0-6)$ & \\
\hline \multirow[t]{2}{*}{ Annese et al. [16] } & $8 \mathrm{PD}$ & NA & NA & NA & NA & NA & NA \\
\hline & 8 BoTx & & & NA & & & \\
\hline \multirow[t]{2}{*}{ Ghoshal et al. [17] } & $10 \mathrm{PD}$ & $34.3 \pm 9.0$ & 50 & $42.6 \pm 49.2$ & NA & NA & $36.0 \pm 12.8$ \\
\hline & 7 BoTx & $42.4 \pm 22.3$ & 71.43 & $107 \pm 102$ & NA & NA & $34.3 \pm 16.8$ \\
\hline \multirow[t]{2}{*}{ Mikaeli et al. [18] } & 20 BoTx & $59.8 \pm 11.5^{\mathrm{a}}$ & 40 & $6.9 \pm 6.2$ & $8.6 \pm 2.2^{\mathrm{a}}$ & $2.2 \pm 0.2$ & 12 \\
\hline & $20 \mathrm{PD}$ & $50.1 \pm 8.9$ & 52.6 & $5.9 \pm 7.7$ & $8.3 \pm 2.2$ & $1.2 \pm 0.2$ & \\
\hline \multirow[t]{2}{*}{ Bansal et al. [19] } & $18 \mathrm{PD}$ & $51.8 \pm 3.4$ & 66.67 & $33.6 \pm 10.2$ & $6.0 \pm 0.5$ & $1.6 \pm 0.7$ & $13(3-23)$ \\
\hline & 16 BoTx & $49.8 \pm 3.7$ & 43.75 & $23.4 \pm 5.1$ & $5.8 \pm 0.5$ & $1.9 \pm 0.6$ & \\
\hline \multirow[t]{2}{*}{ Mikaeli et al. [20] } & $27 \mathrm{PD}$ & $30(24-45)$ & 46.15 & NA & $8(6-10)$ & NA & 12 \\
\hline & $27 \mathrm{PD}+\mathrm{BoTx}$ & $38(26-49)$ & 61.54 & NA & $9(6-10)$ & & \\
\hline
\end{tabular}

Endoscopic myotomy: different treatment methods compared with laparoscopic myotomy

\begin{tabular}{|c|c|c|c|c|c|c|c|}
\hline \multirow[t]{2}{*}{ Zaninotto et al. [21] } & 40 BoTx & $55(19-73)$ & 55 & $18(2-240)$ & $17(8-22)$ & 2.04 & $23(12-34)$ \\
\hline & $40 \mathrm{LM}$ & $48(18-71)$ & 55 & $24(2-240)$ & $18(6-22)$ & 3.24 & \\
\hline \multirow[t]{2}{*}{ Zaninotto et al. [22] } & $20 \mathrm{LM}$ & $47(20-66)$ & 60 & $24(2-20)$ & $17(9-22)$ & NA & $17(12-34)$ \\
\hline & 17 BoTx & $40(19-73)$ & 64.71 & $26(3-240)$ & $17(12-22)$ & & \\
\hline \multirow[t]{2}{*}{ Suárez et al. [23] } & $14 \mathrm{LM}$ & $39.5(17-70)$ & 42.76 & $17.5(2-48)$ & $12(0-19)$ & NA & NA \\
\hline & $16 \mathrm{PD}$ & $43.2(16-71)$ & 37.5 & $22.7(2-60)$ & $8.5(3-18)$ & & \\
\hline \multirow[t]{2}{*}{ Kostic et al. [24] } & $26 \mathrm{PD}$ & $45 \pm 18(20-78)$ & 50 & $18(2-120)$ & NA & NA & 12 \\
\hline & $25 \mathrm{LM}$ & $43 \pm 14(17-66)$ & 44 & $20(8-106)$ & & & \\
\hline \multirow[t]{2}{*}{ Patti et al. [25] } & $35 \mathrm{TM}$ & $45(11-96)$ & 57.14 & NA & NA & NA & 28 \\
\hline & 133 LM + Dor & & & & & & 23 \\
\hline \multirow[t]{2}{*}{ Pasricha et al. [26] } & 11 BoTx & $57(19-72)$ & 45.45 & $36(9-364)$ & $7.1 \pm 1.2^{\mathrm{a}}$ & $1.6 \pm 2.2^{\mathrm{a}}$ & $9(7-16)$ \\
\hline & 10 Placebo & $54(22-74)$ & 60 & $47(8-120)$ & $5.9 \pm 1.6$ & $5.4 \pm 2.0$ & \\
\hline \multirow[t]{2}{*}{ Yi et al. [27] } & $21 \mathrm{FGBD}$ & $39(21-72)$ & 47.62 & NA & NA & NA & NA \\
\hline & $37 \mathrm{PD}$ & $22(9-77)$ & 51.35 & NA & & & \\
\hline \multirow[t]{2}{*}{ Mearin et al. [28] } & $17 \mathrm{PD}$ & $52(5)$ & 52.94 & $49(9)$ & NA & NA & 12 \\
\hline & $24 \mathrm{MD}$ & $50(2)$ & 66.67 & $48(8)$ & NA & NA & \\
\hline \multirow[t]{2}{*}{ Csendes et al. [29] } & $39 \mathrm{PD}$ & $40.2(16-73)$ & 41.1 & $58(24-144)$ & NA & NA & $18(24-44)$ \\
\hline & $40 \mathrm{EC}$ & $42.7(15-80)$ & 47.62 & $62(26-156)$ & NA & NA & \\
\hline \multirow[t]{2}{*}{ Richards et al. [30] } & 21 Heller & $50(24-76)$ & 47.62 & NA & $9(5-10)$ & NA & \\
\hline & 22 Heller + Dor & $50(22-73)$ & 54.55 & NA & $8(5-10)$ & & \\
\hline
\end{tabular}

$N A$ no analysis, PD pneumatic dilatation, BoTx botulinum toxin injection, LM laparoscopic myotomy, Heller Heller myotomy, Heller + Dor Heller myotomy with Dor fundoplication, $T M$ thoracoscopic myotomy, $F G B D$ fluoroscopically guided balloon dilation, $M D$ metallic dilation, $E C$ esophagectomy

a Mean \pm standard deviation $(\mathrm{SD})$ 